\section{Legg-Calvé-Perthes disease in a child with osteopetrosis}

\author{
Alex L. Sims, Thomas W. Barwick, \\ Richard J. Montgomery \\ The James Cook University Hospital, \\ Middlesbrough, UK
}

\begin{abstract}
Osteopetrosis is a rare inherited disorder of bone causing increased bone density. LeggCalvé-Perthes disease (LCPD), by contrast, is a more common idiopathic condition leading to variable avascular necrosis of the immature femoral head. We present a case of a 5 -year-old boy presenting with these co-morbidities. We have found only one previous reference suggesting these two conditions can coexist in the literature. We discuss the basic principles of management of this interesting case.
\end{abstract}

\section{Introduction}

Osteopetrosis is a rare, inherited disorder of bone, leading to increased bone density. There are varying patterns of heritability and also variation in penetrance. Hence incidence is estimated at 1:250,000 for autosomal recessive and 1:20,000 for autosomal dominant inherited variations. The aetiology of osteopetrosis is one of failure of osteoclast function leading to marble bone. The clinical manifestations are particularly variable, with the milder forms being identified as incidental findings on radiograph and the more severe forms causing stunted growth, deformity, recurrent infections, anaemia and compensatory hepatosplenomegaly. Fractures are more common due to dysfunction of the bone-remodelling unit resulting in brittle bone. ${ }^{1}$

Legg-Calvé-Perthes disease (LCPD), by contrast, is an idiopathic condition leading to variable avascular necrosis of the immature femoral head. This is a more common condition with a lifetime risk of 1:1000. The condition is self-limiting although osteoarthritis of the hip may subsequently develop as consequence of head and acetabular incongruity. ${ }^{2}$ In one study of conservatively managed Perthes disease, radiographic changes consistent with osteoarthritis were identified in eighteen of a cohort of sixty hips (30\%) at 34 years following original presentation.3 Thus long term follow up is an important part of management of this condition.

This is the first case report to examine the presentation of a child with LCPD in the presence of osteopetrosis. We have found only one previous reference suggesting these two conditions can coexist in the literature. ${ }^{4}$ We discuss the basic principles of management of this interesting case.

\section{Case Report}

A five-year-old boy presented to the paediatric orthopaedic outpatient clinic with a 1year history of a mild right-sided limp of insidious onset. There was no history of previous limb fracture. The child was known to have osteopetrosis with no concerns in his developmental history. There was a positive family history of osteopetrosis on the maternal side. There was no evidence of thrombophilic tendency nor systemic complications as a result of osteopetrosis at presentation.

On examination he had a mild Trendelenberg gait and a globally reduced range of motion of the right hip. Flexion of $20^{\circ}$, extension of $0^{\circ}$, abduction of $15^{\circ}$, internal rotation of $5^{\circ}$ and external rotation of $20^{\circ}$ was demonstrated. His left hip was found to have a normal range of movement. No evidence of scoliosis or significant limb length discrepancy was identified.

Plain radiographs demonstrated fragmentation of the right capital femoral epiphysis (Figure 1). A magnetic resonance imaging (MRI) scan confirmed an enlarged flattened femoral head with expansion of the right proximal femoral metaphysis, loss of joint space, and flattening of the superior acetabular margin, in keeping with a diagnosis of LCPD (Figures 2 and 3) MRI was important to confirm LCPD appearances within the hip as osteopetrosis may also cause epiphyseal irregularities that might appear similar on radiographs.

In view of his restricted range of movement he was treated as an in-patient with skin traction in abduction and physiotherapy. Within two weeks, flexion of $120^{\circ}$, extension of $5^{\circ}$, abduction $40^{\circ}$, internal rotation of $15^{\circ}$, external rotation of $45^{\circ}$ and adduction of $20^{\circ}$ was achieved. Hydrotherapy and physiotherapy continued in the community as the LCPD process continues.

\section{Discussion}

The patient was less than 5 years old when his LCPD symptoms commenced and in this age group the development of a congruent pain free hip is more likely. However, one must be mindful that the presence of osteopetrosis theoretically increases the risk of future avascular episodes. Intermediate and autosomal dominant forms of osteopetrosis are known to
Correspondence: Alex L. Sims, The James Cook University Hospital, Marton Road, Middlebrough. UK, TS4 3BW.

E-mail: asims@nhs.net

Key words: Legg-Calvé-Perthes, Perthes, osteopetrosis.

Received for publication: 8 November 2011.

Revision received: 26 November 2011.

Accepted for publication: 30 November 2011

This work is licensed under a Creative Commons Attribution NonCommercial 3.0 License (CC BYNC 3.0).

(C) Copyright A.L. Sims et al., 2011

Licensee PAGEPress, Italy

Clinics and Practice 2011; 1:e139

doi:10.4081/cp.2011.e139

exhibit skeletal manifestations including increased frequency of fracture, osteomyelitis and bowing of long bones. ${ }^{5}$ Certainly the predisposal to fracture and the development of osteomyelitis could have damaging effects on the blood supply to the proximal femur, causing avascular necrosis. Also, one could postulate that osteopetrosis has the continued capacity to reduce the femoral head blood supply either directly, by interrupting or reducing the number of vessels reaching the epiphysis or indirectly, via the presence of higher intraosseous pressures in the femoral head reducing blood flow to it, leading to osteonecrosis. ${ }^{6}$

A recent review, based on animal studies, has suggested that use of bisphosphonates and other anticatabolic agents in patients with LCPD may reduce bone resorption and protect against collapse and deformation of the femoral head. ${ }^{7}$ As osteoclastic activity is inhibited in osteopetrosis, thereby reducing catabolism, it might be expected that osteopetrosis protects from the effects of LCPD. However, in this case report, head collapse still occurred and so further clinical studies of LCPD in the presence of normal bone remodelling unit function are needed.

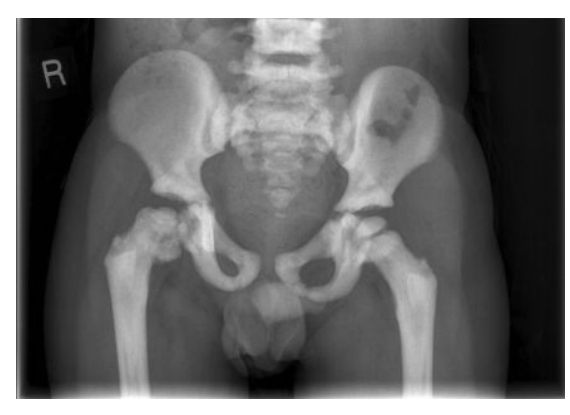

Figure 1. Anteroposterior radiograph to demonstrate fragmentation of the right capital femoral epiphysis. 
The treatment of Perthes disease is controversial and includes both conservative and operative measures. The aim is to reduce pain and contain the damaged femoral head within the acetabulum whilst the re-ossification and

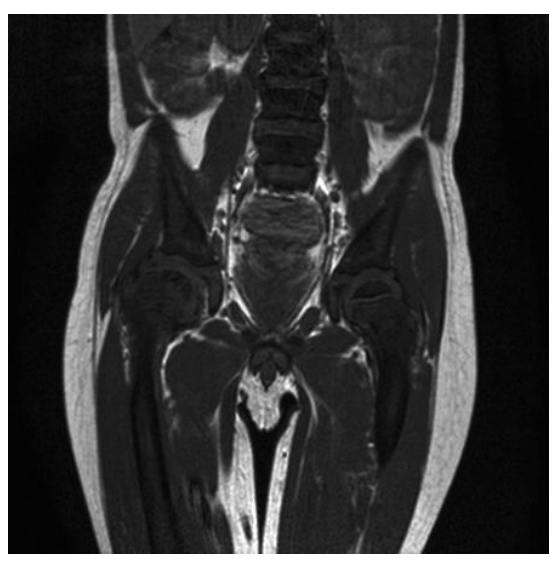

Figure 2. Coronal view of magnetic resonance imaging.

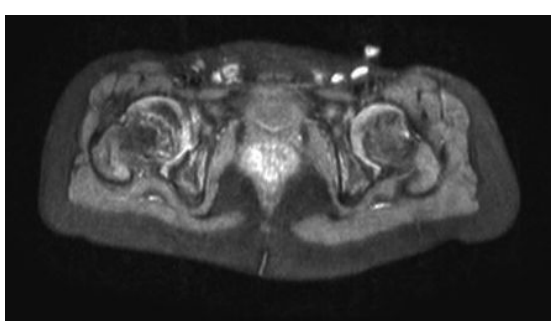

Figure 3. Axial view of magnetic resonance imaging. healing phases occur, usually over a $3-5$ year period. Maintenance of abduction range is thought to assist in the acetabular moulding of the damaged immature femoral head and prevent extrusion and further collapse. Increased range of motion in all planes is essential for nourishment of cartilage by increased synovial fluid production. Hence, conservative management options include analgesia, activity modification, use of crutches or wheelchair use, range of motion improvement with physiotherapy/hydrotherapy, and traction or abduction bracing. Operative treatments include femoral, pelvic or combined osteotomy with soft tissue procedures such as adductor tenotomy to afford containment of the hip and increase abduction range. ${ }^{8}$ Should operative intervention ever be considered in the presence of osteopetrosis, complications such as fracture, non-union, malunion and osteomyelitis will be encountered more frequently and must be borne in mind with appropriate counselling for patients and relatives. ${ }^{1}$

\section{Conclusions}

This is the first case report describing the management of a patient with osteopetrosis developing unilateral Perthes disease. Simple conservative measures including traction, analgesia and physiotherapy have significantly improved the range of movement of the hip despite a delay in presentation.

\section{References}

1. Stark Z, Savarirayan R. Osteopetrosis. Orphanet J Rare Dis 2009;4:5.

2. Kim HK. Legg-Calvé-Perthes disease. J Am Acad Orthop Surg 2010;18;676-86.

3. Lecuire F. The long-term outcome of primary osteochondritis of the hip (LeggCalvé-Perthes' disease). J Bone Joint Surg Br 2002;84:636-40.

4. Kaibara N, Katsuki I, Hotokebuchi T, Takagishi K. Intermediate form of osteopetrosis with recessive inheritance. Skelet Radiol 1982;9:47-51.

5. Shapiro, F. Osteopetrosis: Current clinical considerations. Clin Orthop Relat Res 1993;294:34-44.

6. Whyte M, Wenkert D, Clements KL, et al. Bisphosphonate-induced osteopetrosis. N Engl J Med 2003;349:457-63.

7. Little DG, Kim HK. Potential for bisphosphonate treatment in Legg-Calve-Perthes disease. J Pediatr Orthop 2011;31 Suppl 2:S182-8.

8. Hefti F, Clarke NM. The management of Legg-Calvé-Perthes disease: is there a consensus? A study of clinical practice preferred by the members of the European Paediatric Orthopaedic Society. J Child Orthop 2007;1:19-25. 